\title{
Modeling the expected cost-effectiveness and impact of oral pre- exposure prophylaxis in Uganda and Swaziland
}

Project SOAR

Follow this and additional works at: https://knowledgecommons.popcouncil.org/departments_sbsr-hiv

Part of the Demography, Population, and Ecology Commons, Family, Life Course, and Society Commons, International Public Health Commons, and the Women's Health Commons How does access to this work benefit you? Let us know!

\section{Recommended Citation}

Project SOAR. 2018. "Modeling the expected cost-effectiveness and impact of oral pre-exposure prophylaxis in Uganda and Swaziland," Project SOAR Activity Brief. Washington, DC: Population Council. 


\section{Modeling the Expected Cost-effectiveness and Impact of Oral Pre-exposure Prophylaxis in Uganda and Swaziland}

Clinical trials have shown that oral antiretrovirals (ARVs) containing tenofovir/emtricitabine used by HIV-negative individuals to prevent HIV acquisition, called oral pre-exposure prophylaxis (PrEP), are safe and effective in several populations. Oral PrEP is now considered part of the PEPFAR HIV prevention toolkit, and procurement by PEPFAR is permitted in countries where (1) test and start policies have been initiated; (2) viral load testing policies call for testing at least annually; and (3) multi-month ARV provision is available for stable clients.

Prior modeling work has demonstrated that oral PrEP is most cost effective when targeted to populations at very high risk of infection, with risk dependent on a combination of individual behavior and epidemic contexts. Project SOAR is assisting Uganda and Swaziland' in making critical decisions about oral PrEP introduction and scale up by modelling the expected cost-effectiveness

PrEP modelling is also being conducted in Mozambique and Lesotho through Health Policy Plus (HP+).

Research Partners: Avenir Health, Palladium

Location: Swaziland and Uganda

Study Duration: 2017-2018

For more information, contact Steven Forsythe, Avenir Health, sforsythe@ avenirhealth.org.

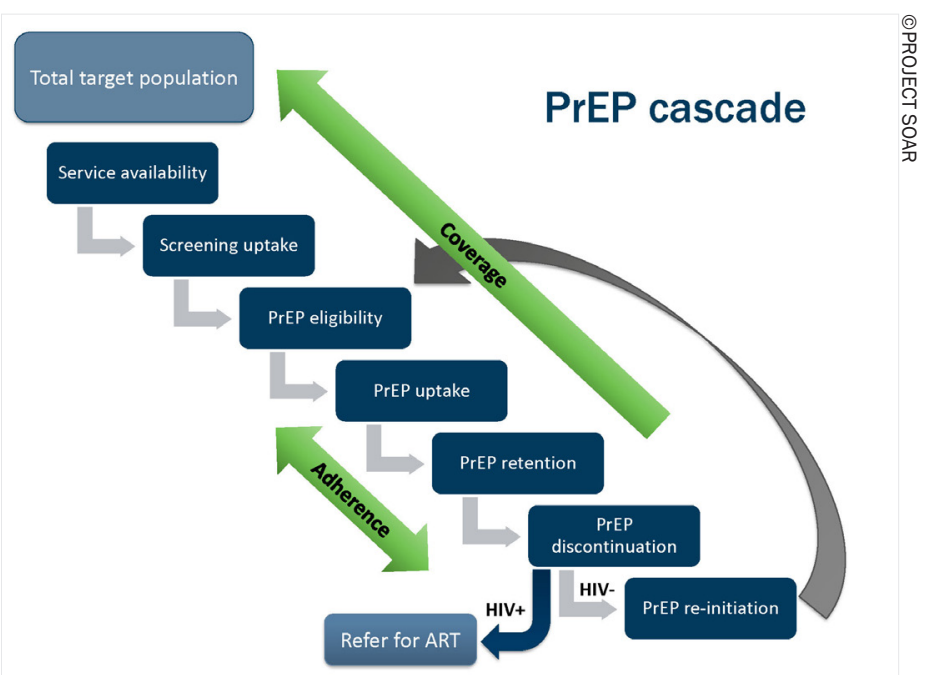

Modelling the expected cost effectiveness and impact of oral PrEP within Uganda and Swaziland's portfolio of HIV interventions will help each country make critical decisions about oral PrEP.

and impact of oral PrEP within each country's larger portfolio of HIV prevention interventions.

\section{OUR RESEARCH}

The Project SOAR team first worked with country stakeholders to identify specific modeling questions that would be helpful to advance PrEP policy and implementation planning and discussions in-country (see box on next page for Uganda's questions). SOAR then adapted, expanded, and applied existing modeling tools to estimate the expected cost-effectiveness and impact of oral PrEP within the larger portfolio of HIV prevention interventions. The modeling tools used are the Goals Model and the Incidence Patterns Model (IPM), which uses published data in Demographic and Health Surveys (DHS), 


\section{What would Uganda like to know from PrEP modelling?}

- How would rolling out oral PrEP to different risk groups and geographic areas affect the impact, cost-effectiveness, and total cost of oral PrEP?

- How do the impact, cost, and cost effectiveness vary by risk group?

- Female sex workers

- Serodiscordant couples

- Men who have sex with men

- People who inject drugs

- Adolescent girls and young women with multiple partners

- How would varying levels of future scaleup of ART and voluntary medical male circumcision affect the impact and costeffectiveness of oral PrEP?

- How would varying unit costs of oral PrEP by risk group affect the relative costeffectiveness of providing PrEP to the different risk groups?

- How would varying levels of adherence by risk group affect the relative impact and cost-effectiveness of providing PrEP to the different risk groups?

Population-based HIV Impact Assessments (PHIA) and AIDS Indicator Surveys (AIS) to estimate HIV incidence in each risk group and province. These two models are then combined into a single platform to create the Oral PrEP Workbook-a Microsoft Excel-based tool that links the IPM with Goals and visualizes the results to address policy questions raised in each country.

\section{Strengths of our modelling approach}

- Combines the more detailed risk structure of IPM with the dynamic projections and HIV interventions in the Goals Model.

- Incorporates risk groups not previously modeled in Goals: serodiscordant couples, adolescent girls and young women.

- Dynamic modeling can take into account projected changes in HIV incidence from scaling up ART and other HIV prevention interventions.

- Use of rigorous data from published DHS, PHIA and AIS surveys to disaggregate HIV incidence by risk group.

- Oral PrEP Workbook allows users flexibility in defining rates and levels of scale-up of PrEP, target populations, unit costs, etc. while automating the communication between the IPM and Goals Model.

\section{RESEARCH UTILIZATION}

SOAR, in consultation with the USAID Missions, is presenting their analytical findings in-country to stakeholders within the Ministries of Health and National AIDS Control Programs in Swaziland and Uganda. The purpose of these meetings is to discuss the implications of PrEP in each country and design a strategy for moving forward. agreement funded by the President's Emergency Plan for AIDS Relief and the U. S. Agency for International Development (Agreement No. AIDOAA-A-14-00060). SOAR is able to accept funding from all USAID accounts.
Project SOAR/Population Council

4301 Connecticut Avenue, NW, Suite 280

Washington, DC 20008

Tel: +12022379400

e-mail: ProjectSOAR@popcouncil.org

projsoar.org

(C)Population Council, January 2018 